\title{
About the convergence type of improper integrals defining fractional derivatives
}

\author{
B. Kalam And G. VAinikKo
}

\begin{abstract}
This article continues the analysis of the class of fractionally differentiable functions. We complete the main result of [4] that characterises the class of fractionally differentiable functions in terms of the pointwise convergence of certain improper integrals containing these functions. Our aim is to present an example, which shows that in order to obtain all fractionally differentiable functions, one may not replace the conditional convergence of those integrals by their absolute convergence.
\end{abstract}

\section{Introduction}

Consider the Riemann-Liouville integral operator $J^{\alpha}: C[0, T] \rightarrow C[0, T]$ of order $\alpha>0, \alpha \in \mathbb{R}$, defined by

$$
\left(J^{\alpha} u\right)(t)=\frac{1}{\Gamma(\alpha)} \int_{0}^{t}(t-s)^{\alpha-1} u(s) d s, \quad 0 \leq t \leq T, \quad u \in C[0, T],
$$

where $\Gamma$ is the Euler gamma-function. In particular, $\left(J^{1} u\right)(t)=\int_{0}^{t} u(s) d s$. It is well known that, for $\alpha=m \in \mathbb{N}=\{1,2, \ldots\}$, the range of the operator $J^{m}$ is given by

$$
J^{m} C[0, T]=\left\{v \in C^{m}[0, T]: v^{(k)}(0)=0, k=0, \ldots, m-1\right\}=: C_{0}^{m}[0, T],
$$

and $J^{m}$ is invertible on it, $\left(J^{m}\right)^{-1} v=D_{0}^{m} v$, where $D_{0}^{m}: C_{0}^{m}[0, T] \rightarrow C[0, T]$ is the restriction of the operator $D^{m}=(d / d t)^{m}: C^{m}[0, T] \rightarrow C[0, T]$. Due to the semigroup property (see, e.g., $[2,1]$ ),

$$
J^{\alpha} J^{\beta}=J^{\beta} J^{\alpha}=J^{\alpha+\beta} \quad \text { for } \quad \alpha>0, \beta>0,
$$

and $J^{\alpha} u$ can be interpreted as a fractional integral of $u$. Note that $J^{\alpha}$ is invertible on its range $J^{\alpha} C[0, T]$ also for fractional (noninteger) $\alpha>0$.

Received July 24, 2018.

2010 Mathematics Subject Classification. 26A33.

Key words and phrases. Fractionally differentiable functions; convergence of improper integrals; fractional derivatives.

http://dx.doi.org/10.12697/ACUTM.2019.23.10 
Indeed, if $J^{\alpha} u=0$ for some $u \in C[0, T]$, then, taking $m \in \mathbb{N}, m>\alpha$, we have $u=0$, since

$$
J^{m} u=J^{m-\alpha} J^{\alpha} u=0 .
$$

The description of the range $J^{\alpha} C[0, T], \alpha>0$, is closely related to the description of the class of fractionally differentiable functions. Namely, one possible definition of the fractional differentiation operator of order $\alpha>0$ is given by

$$
D_{0}^{\alpha} v=\left(J^{\alpha}\right)^{-1} v, \quad v \in J^{\alpha} C[0, T] .
$$

This most natural definition is used, e.g., in the Mathematical Encyclopedia [5]. The more popular Riemann-Liouville and Caputo fractional derivatives $D_{R-L}^{\alpha} v$ and $D_{C a p}^{\alpha} v$ are closely related to $D_{0}^{\alpha} v$, see [4] for details.

By $\mathcal{H}^{\alpha}[0, T], 0<\alpha \leq 1$, we mean the standard Hölder space consisting of functions $v \in C[0, T]$ such that

$$
\|v\|_{\mathcal{H}^{\alpha}}:=\max _{0 \leq t \leq T}|v(t)|+\sup _{0 \leq s<t \leq T} \frac{|v(t)-v(s)|}{(t-s)^{\alpha}}<\infty,
$$

and by $\mathcal{H}_{0}^{\alpha}[0, T], 0<\alpha<1$, we mean the closed (see [3]) subspace of $\mathcal{H}^{\alpha}[0, T]$ consisting of functions $v \in \mathcal{H}^{\alpha}[0, T]$ such that

$$
\lim _{\varepsilon \rightarrow 0} \sup _{0 \leq s<t \leq T, t-s \leq \varepsilon} \frac{|v(t)-v(s)|}{(t-s)^{\alpha}}=0 .
$$

Although the concept of fractionally differentiable functions is old, the class of all fractionally differentiable functions has been described only recently. Below we formulate one possible form of the criteria for fractional differentiability of a function.

Theorem 1 (see [4], Theorem 2.1). A function $v \in C[0, T]$ is $D_{0}^{\alpha}$-differentiale for an $\alpha \in(0,1)$, i.e., $v \in J^{\alpha} C[0, T]$, if and only if $v$ has the structure $v=\gamma_{0} t^{\alpha}+v_{0}$, where $\gamma_{0}$ is a constant, $v_{0} \in \mathcal{H}_{0}^{\alpha}[0, T], v(0)=0$, and the improper integral

$$
w(t):=\int_{0}^{t}(t-s)^{-\alpha-1}(v(t)-v(s)) d s, \quad 0<t \leq T
$$

converges for any $t \in(0, T]$ and defines a function $w \in C(0, T]$, which has a finite limit $w(0):=\lim _{t \rightarrow 0} w(t)$. Besides, for $0<t \leq T$,

$$
\begin{gathered}
\left.\left(D_{0}^{\alpha} v\right)(t)\right)=\frac{1}{\Gamma(1-\alpha)}\left(t^{-\alpha} v(t)+\alpha \int_{0}^{t}(t-s)^{-\alpha-1}(v(t)-v(s)) d s\right), \\
\left(D_{0}^{\alpha} v\right)(0):=\left(\left(J^{\alpha}\right)^{-1} v\right)(0)=\Gamma(\alpha+1) \gamma_{0} .
\end{gathered}
$$

The situation seems to be similar for the fractional differentiability of a function $v \in L^{p}(0, T)$. According to Conjecture 7.1 of [4], a function $v \in L^{p}(0, T), 1<p \leq \infty$, belongs to $J^{\alpha} L^{p}(0, T), 0<\alpha<1$, if and only if $t^{-\alpha} v(t)$ lies in $L^{p}(0, T)$, the integral (1) is well defined for almost 
every $t \in(0, T]$ in the Lebesgue sense (hence converges absolutely), and $w \in L^{p}(0, T)$. An essential question arrives: whether in Theorem 1 one can replace the conditional convergence of the improper integrals (1) by their absolute convergence? The answer occurs to be "no": restricting ourselves to the absolute convergence of those integrals, we do not obtain all functions $v \in J^{\alpha} C[0, T]$. To prove this claim, we construct an example function $v \in J^{\alpha} C[0, T]$, such that the integral (1) does not converge absolutely for some $t \in(0, T]$. Namely, as we shall see in Section 2,

$v(t)=t(1-t)^{\alpha}(\log (1-t))^{-1} \sin (\log (1-t)), \quad 0<t<1, \quad v(0)=v(1)=0$,

occurs to be such a function with $T=1$.

\section{The study of the example function}

To see the continuity of $v(t)$ defined in (2) at points $t=0$ and $t=T=1$, we observe that

$\lim _{t \rightarrow 0} v(t)=\lim _{t \rightarrow 0} \frac{t(1-t)^{\alpha} \sin (\log (1-t))}{\log (1-t)}=\lim _{t \rightarrow 0} t(1-t)^{\alpha} \cdot \lim _{t \rightarrow 0} \frac{\sin (\log (1-t))}{\log (1-t)}=0$

and

$\lim _{t \rightarrow 1} v(t)=\lim _{t \rightarrow 1} \frac{t(1-t)^{\alpha} \sin (\log (1-t))}{\log (1-t)}=\lim _{t \rightarrow 1} t(1-t)^{\alpha} \cdot \lim _{t \rightarrow 1} \frac{\sin (\log (1-t))}{\log (1-t)}=0$.

Proposition 1. The function $v(t)$ defined in (2) satisfies the following conditions of Theorem 1, with $T=1$ and $\gamma_{0}=0$ :

$$
\begin{gathered}
v \in \mathcal{H}_{0}^{\alpha}[0,1], \\
w(t):=\int_{0}^{t}(t-s)^{-\alpha-1}(v(t)-v(s)) d s \text { converges for any } t \in(0,1], \\
w \in C(0,1] \text { and } \lim _{t \rightarrow 0} w(t)=0 \text {, thus } w \in C[0,1] .
\end{gathered}
$$

By Theorem 1, $v \in J^{\alpha} C[0,1] \subset J^{\alpha} L^{p}(0, T), p \leq 1$, so $v$ is $D_{0}^{\alpha}$-differentiable in the spaces $C[0,1]$ and $L^{p}(0,1)$. We claim that for $t=1$ the convergence of the integral (4) is non-absolute:

$$
\begin{aligned}
& \int_{0}^{1}(1-s)^{-\alpha-1}|v(1)-v(s)| d s \\
& =\int_{0}^{1} s(1-s)^{-1}|\log (1-s)|^{-1}|\sin (\log (1-s))| d s=\infty .
\end{aligned}
$$

Proof. Proof of (3). First we show that $v \in C^{1}[0,1)$. One has

$$
v^{\prime}(t)=\sum_{i=1}^{4} u_{i}(t)
$$


where

$$
\begin{aligned}
& u_{1}(t)=(1-t)^{\alpha}(\log (1-t))^{-1} \sin (\log (1-t)), \\
& u_{2}(t)=-\alpha \cdot t(1-t)^{\alpha-1}(\log (1-t))^{-1} \sin (\log (1-t)), \\
& u_{3}(t)=t(1-t)^{\alpha-1}(\log (1-t))^{-2} \sin (\log (1-t)), \\
& u_{4}(t)=-t(1-t)^{\alpha-1}(\log (1-t))^{-1} \cos (\log (1-t)) .
\end{aligned}
$$

Clearly $v^{\prime} \in C(0,1)$. To show that $v \in C^{1}[0,1)$ we have to notice that a finite limit $\lim _{t \rightarrow 0} u_{i}(t)$ exists for $i=1,2,3,4$. Using the change of variable $\log (1-t)=x$, we get

$$
\lim _{t \rightarrow 0} \frac{\sin (\log (1-t))}{\log (1-t)}=\lim _{x \rightarrow 0} \frac{\sin x}{x}=1 .
$$

Knowing this, let us analyse the limits:

$$
\begin{aligned}
\lim _{t \rightarrow 0} u_{1}(t)=\lim _{t \rightarrow 0} \frac{(1-t)^{\alpha} \sin (\log (1-t))}{\log (1-t)}=\lim _{t \rightarrow 0}(1-t)^{\alpha} \cdot \lim _{t \rightarrow 0} \frac{\sin (\log (1-t))}{\log (1-t)}=1, & \lim _{t \rightarrow 0} u_{2}(t)=\lim _{t \rightarrow 0} \frac{-\alpha t(1-t)^{\alpha-1} \sin (\log (1-t))}{\log (1-t)} \\
& =-\alpha \lim _{t \rightarrow 0} t(1-t)^{\alpha-1} \cdot \lim _{t \rightarrow 0} \frac{\sin (\log (1-t))}{\log (1-t)}=0, \\
\lim _{t \rightarrow 0} u_{3}(t) & =\lim _{t \rightarrow 0} \frac{t(1-t)^{\alpha-1} \sin (\log (1-t))}{(\log (1-t))^{2}}= \\
& =\lim _{t \rightarrow 0} \frac{\sin (\log (1-t))}{\log (1-t)} \cdot \lim _{t \rightarrow 0}(1-t)^{\alpha-1} \cdot \lim _{t \rightarrow 0} \frac{t}{\log (1-t)} \\
& =\lim _{t \rightarrow 0} \frac{t}{\log (1-t)}=\lim _{t \rightarrow 0} \frac{1}{-\frac{1}{1-t}}=-1 \\
\lim _{t \rightarrow 0} u_{4}(t) & =\lim _{t \rightarrow 0} \frac{-t(1-t)^{\alpha-1} \cos (\log (1-t))}{\log (1-t)} \\
& =\lim _{t \rightarrow 0} \frac{-t}{\log (1-t)} \cdot \lim _{t \rightarrow 0}(1-t)^{\alpha-1} \cdot \lim _{t \rightarrow 0} \cos (\log (1-t) \\
& =\lim _{t \rightarrow 0} \frac{-t}{\log (1-t)}=1 .
\end{aligned}
$$

Thus, $v^{\prime}(0):=\lim _{t \rightarrow 0} v^{\prime}(t)=1+0-1+1=1$, and $v \in C^{1}[0,1)$.

Further, observe that

$$
\lim _{t \rightarrow 1}(1-t)^{1-\alpha} v^{\prime}(t)=\lim _{t \rightarrow 1} \sum_{i=1}^{4}(1-t)^{1-\alpha} u_{i}(t)=0 .
$$


Indeed, in the following fractions the numerator is bounded and the denominator tends to infinity:

$$
\begin{aligned}
& \lim _{t \rightarrow 1}(1-t)^{1-\alpha} u_{1}(t)=\lim _{t \rightarrow 1} \frac{(1-t) \sin (\log (1-t))}{\log (1-t)}=0, \\
& \lim _{t \rightarrow 1}(1-t)^{1-\alpha} u_{2}(t)=\lim _{t \rightarrow 1} \frac{-\alpha t \sin (\log (1-t))}{\log (1-t)}=0 \\
& \lim _{t \rightarrow 1}(1-t)^{1-\alpha} u_{3}(t)=\lim _{t \rightarrow 1} \frac{t \sin (\log (1-t))}{(\log (1-t))^{2}}=0 \\
& \lim _{t \rightarrow 1}(1-t)^{1-\alpha} u_{4}(t)=\lim _{t \rightarrow 1} \frac{-t \cos (\log (1-t))}{\log (1-t)}=0 .
\end{aligned}
$$

We also conclude that $(1-t)^{1-\alpha}\left|v^{\prime}(t)\right||\log (1-t)| \leq c, 0 \leq t<1$, for a constant $c$, and therefore

$$
\left|v^{\prime}(t)\right| \leq c \frac{(1-t)^{\alpha-1}}{|\log (1-t)|}, \quad 0 \leq t<1
$$

We are ready to finish the proof of (3). Since $v \in C^{1}[0,1)$, we have $v \in \mathcal{H}_{0}^{\alpha}[0, \theta]$ for all $\theta \in(0,1)$. So it remains to show that

$$
0 \leq s_{n}<t_{n}<1 \quad \text { and } s_{n}, t_{n} \rightarrow 1 \Rightarrow \frac{v\left(t_{n}\right)-v\left(s_{n}\right)}{\left(t_{n}-s_{n}\right)^{\alpha}} \rightarrow 0 \text { as } n \rightarrow \infty \text {. }
$$

We estimate $\left(\right.$ for $\left.s_{n}, t_{n} \rightarrow 1\right)$ :

$$
\begin{aligned}
\left|v\left(t_{n}\right)-v\left(s_{n}\right)\right| & =\left|\int_{s_{n}}^{t_{n}} v^{\prime}(\tau) d \tau\right|=\left|\int_{s_{n}}^{t_{n}}(1-\tau)^{\alpha-1}(1-\tau)^{1-\alpha} v^{\prime}(\tau) d \tau\right| \\
& \leq \max _{s_{n} \leq \tau \leq t_{n}}\left((1-\tau)^{1-\alpha}\left|v^{\prime}(\tau)\right|\right) \int_{s_{n}}^{t_{n}}(1-\tau)^{\alpha-1} d \tau
\end{aligned}
$$

Here

$$
\int_{s_{n}}^{t_{n}}(1-\tau)^{\alpha-1} d \tau=\frac{1}{\alpha}\left(\left(1-s_{n}\right)^{\alpha}-\left(1-t_{n}\right)^{\alpha}\right) \leq \frac{1}{\alpha}\left(t_{n}-s_{n}\right)^{\alpha},
$$

since $\left|t^{\alpha}-s^{\alpha}\right| \leq|t-s|^{\alpha}$, for $0<\alpha<1, s, t \geq 0$. Applying (9), we obtain

$$
\frac{\left|v\left(t_{n}\right)-v\left(s_{n}\right)\right|}{\left(t_{n}-s_{n}\right)^{\alpha}} \leq \frac{1}{\alpha} \max _{s_{n} \leq \tau \leq t_{n}}(1-\tau)^{1-\alpha}\left|v^{\prime}(\tau)\right| \rightarrow 0 .
$$

Thus $v \in \mathcal{H}_{0}^{\alpha}[0,1]$.

Proof of (4). For $0<t<1$, integrating by parts gives

$$
w(t)=\int_{0}^{t}(t-s)^{-\alpha-1}(v(t)-v(s)) d s=-\frac{1}{\alpha} t^{-\alpha} v(t)+\frac{1}{\alpha} \int_{0}^{t}(t-s)^{-\alpha} v^{\prime}(s) d s .
$$


Since $v \in \mathcal{H}_{0}^{\alpha}[0,1]$ and $v(0)=0$, we have

$$
t^{-\alpha} v(t)=\frac{v(t)-v(0)}{t^{\alpha}} \rightarrow 0, \quad \text { as } t \rightarrow 0
$$

With this limit value at $t=0$ the function $t^{-\alpha} v(t)$ is continuous in $[0,1]$. The integral in the right hand side of (11) converges for $0<t<1$, since $v \in C^{1}[0,1)$. Moreover, we observe that $w \in C[0,1), w(0)=0$. Let now $t=1$. We prove that also the improper integral $\int_{0}^{1}(1-s)^{-\alpha} v^{\prime}(s) d x$ converges. According to (7) we present this integral as the sum of four integrals. Making the change of variables $\log (1-s)=-x$, we have $s=1-e^{-x}, d s=e^{-x} d x$, and we get

$$
\begin{aligned}
& \int_{0}^{1}(1-s)^{-\alpha} u_{1}(s) d s=\int_{0}^{1}(\log (1-s))^{-1} \sin (\log (1-s)) d s \\
& =\int_{0}^{\infty} \frac{e^{-x}}{-x} \sin (-x) d x=\int_{0}^{\infty} \frac{e^{-x} \sin x}{x} d x \\
& =\int_{0}^{\infty} f_{1}(x) \sin x d x, \quad f_{1}(x)=\frac{e^{-x}}{x} \\
& \int_{0}^{1}(1-s)^{-\alpha} u_{2}(s) d s=-\alpha \int_{0}^{1} s(1-s)^{-1}(\log (1-s))^{-1} \sin (\log (1-s)) d s \\
& =-\alpha \int_{0}^{\infty} e^{x}\left(1-e^{-x}\right) \cdot \frac{e^{-x}}{-x} \cdot \sin (-x) d x \\
& =-\alpha \int_{0}^{\infty} \frac{1-e^{-x}}{x} \sin x d x \\
& =\int_{0}^{\infty} f_{2}(x) \sin x d x, \quad f_{2}(x)=\frac{1-e^{-x}}{x} \\
& \int_{0}^{1}(1-s)^{-\alpha} u_{3}(s) d s=\int_{0}^{1} s(1-s)^{-1}(\log (1-s))^{-2} \sin (\log (1-s)) d s \\
& =\int_{0}^{\infty}\left(1-e^{-x}\right) e^{x}(-x)^{-2} e^{-x} \sin (-x) d x \\
& =-\int_{0}^{\infty} \frac{1-e^{-x}}{x^{2}} \sin x d x \\
& =-\int_{0}^{\infty} f_{3}(x) \sin x d x, \quad f_{3}(x)=-\frac{1-e^{-x}}{x^{2}}
\end{aligned}
$$




$$
\begin{aligned}
\int_{0}^{1}(1-s)^{-\alpha} u_{4}(s) d s & =\int_{0}^{1} s(1-s)^{-1}(\log (1-s))^{-1} \cos (\log (1-s)) d s \\
& =\int_{0}^{\infty}\left(1-e^{-x}\right) e^{x} \cdot \frac{e^{-x}}{-x} \cdot \cos (-x) d x \\
& =-\int_{0}^{\infty} \frac{1-e^{-x}}{x} \cos x d x \\
& =\int_{0}^{\infty} f_{4}(x) \cos x d x, \quad f_{4}(x)=-\frac{1-e^{-x}}{x} .
\end{aligned}
$$

Clearly $f_{2}, f_{4} \in C[0, \infty)$, and $f_{1}(x) \sin x$ and $f_{3}(x) \sin x$ are in $C[0, \infty)$. Due to Dirichlet's test, integrals $\int_{0}^{\infty} f(x) \sin x d x$ and $\int_{0}^{\infty} f(x) \cos x d x$ converge if function $f(x)$ tends monotonically to 0 as $x \rightarrow \infty$, and this is the case for $f_{i}(x), i=1,2,3,4$. Thus the integrals $\int_{0}^{\infty} f_{i}(x) \sin x d x, i=1,2,3,4$, converge and hence (4) holds true also for $t=1$.

Proof of (5). In the proof of (4) we already saw that $w \in C[0,1)$. It remains to prove the continuity of $w$ at $t=1$, i.e.,

$$
\int_{0}^{t}(t-s)^{-\alpha} v^{\prime}(s) d s \rightarrow \int_{0}^{1}(1-s)^{-\alpha} v^{\prime}(s) d s \text { as } t \rightarrow 1 .
$$

We have already shown that the improper integral $\int_{0}^{1}(1-s)^{\alpha} v^{\prime}(s) d s$ converges, hence $\int_{t}^{1}(1-s)^{\alpha} v^{\prime}(s) d s \rightarrow 0$ as $t \rightarrow 0$. Therefore it is sufficient to show that

$$
\left|\int_{0}^{t}\left[(t-s)^{-\alpha}-(1-s)^{-\alpha}\right] v^{\prime}(s) d s\right| \rightarrow 0 \quad \text { as } \quad t \uparrow 1 .
$$

We establish a more strong relation

$$
\int_{0}^{t}\left[(t-s)^{-\alpha}-(1-s)^{-\alpha}\right] d s \max _{0 \leq s \leq t}\left|v^{\prime}(s)\right| \rightarrow 0 \quad \text { as } \quad t \uparrow 1 .
$$

First,

$$
\begin{aligned}
\int_{0}^{t}\left[(t-s)^{-\alpha}-(1-s)^{-\alpha}\right] d s & =\frac{1}{1-\alpha}\left[(1-t)^{1-\alpha}-1+t^{1-\alpha}\right] \\
& \leq \frac{1}{1-\alpha}(1-t)^{1-\alpha}
\end{aligned}
$$

Further, the function $(1-s)^{\alpha-1}|\log (1-s)|^{-1}$ increases in $(0,1)$, and $(10)$ implies that

$$
\max _{0 \leq s \leq t}\left|v^{\prime}(s)\right| \leq \frac{c(1-t)^{\alpha-1}}{|\log (1-t)|} .
$$

Together with (13), this implies (12), and $w \in C[0,1]$. 
Proof of (6). The integrand in (6) has a singularity only at $s=1$. It is sufficient to analyse the integral

$$
I=\int_{1 / 2}^{1}(1-s)^{-1}|\log (1-s)|^{-1}|\sin (\log (1-s))| d s .
$$

Making the change in variables $\tilde{s}=1-s$ and after that writing again $s$ instead of $\tilde{s}$, we have

$$
I=\int_{0}^{1 / 2} s^{-1}|\log s|^{-1}|\sin (\log s)| d s .
$$

After the change of variable $t=-\log s$, we get that

$$
I=-\int_{\log (2)}^{\infty} e^{t} \cdot t^{-1}|\sin t| e^{-t} d t=\int_{\log (2)}^{\infty} \frac{|\sin t|}{t} d t=\infty .
$$

The proof of Proposition 1 is complete.

\section{Acknowledgment}

This work was partially supported by institutional research funding IUT2057 of the Estonian Ministry of Education and Research.

\section{References}

[1] K. Diethelm, The Analysis of Fractional Differential Equations. An ApplicationOriented Exposition Using Differential Operators of Caputo Type, Lecture Notes in Mathematics 2004, Springer-Verlag, Berlin, 2010.

[2] A. A. Kilbas, H. M. Srivastava, and J. J. Trujillo, Theory and Applications of Fractional Differential Equations, North Holland Mathematics Studies 204, Elsevier, Netherlands, 2006.

[3] G. Vainikko, First kind cordial Volterra integral equations 2, Numer. Funct. Anal. Optim. 35 (2014), 1607-1637.

[4] G. Vainikko, Which functions are fractionally differentiable?, Z. Anal. Anwend. 35 (2016), 465-487.

[5] I. M. Vinogradov et al. (eds.) Mathematical Encyclopedia, Volumes 1-5, Moscow: Soviet Encyclopedia, 1977-1985. (Russian)

Institute of Mathematics and Statistics, University of Tartu, 50409 Tartu, EsTONIA

E-mail address: britt.kalam@ut.ee

E-mail address: gennadi.vainikko@ut.ee 